\title{
Premature Menarche
}

National Cancer Institute

\section{Source}

National Cancer Institute. Premature Menarche. NCI Thesaurus. Code C113338.

Occurrence of the first menstrual period in a female before the usual or expected age. 